\title{
Role of Vitamin D in Dental Health: A Review
}

\author{
Varun Suri', Mohit Bansal², Tarun Kalra ${ }^{3}$
}

\begin{abstract}
Introduction: The role of vitamin D in the development of bones is well known and documented. It also helps in preserving the oral structures, consisting of mainly the teeth and supporting structures. This is the only vitamin which is available through natural sunlight. It is the only vitamin which is found naturally in cod liver oil and butterfat.

Material and methodology: The material presented in this review is a brief summary of the beneficial effects of vitamin $D$ in various conditions, with major emphasis on dental caries, periodontal disease, the genetic basis of vitamin $D$ and positive effect of vitamin $D$ in the immunecompromised population.

Results: Vitamin D is also known as the antirachitic factor as it prevents the occurrence of rickets in adults and osteomalacia in children. However, vitamin $D^{3}$ itself is biologically inert when converted to its active form, is found to be extremely beneficial in various conditions.

Conclusion: This review article focuses on various synergistic effects of vitamin D on oral health by searching a number of published articles from EBSCOhost database.

Keywords: Oral tissues, Teeth, Vitamin D.

Journal of Postgraduate Medicine, Education and Research (2019): 10.5005/jp-journals-10028-1319
\end{abstract}

\section{INTRODUCTION}

ealthy body has a healthy soul. Nutrition has a very important - effect on overall health. Proper functioning of the human body depends upon the form of nutrients taken. Vitamin D, a fat-soluble vitamin has multiple benefits on physical and oral health. The two common sources of vitamin D are diet and sunlight.

Vitamin D has been found to play a major role in the prevention of various types of cancer. ${ }^{1-3}$ Vitamin $D$ is also important for periodontal health and bone metabolism. Fortification of foods with vitamin $D$, especially dairy products is very common in European countries. ${ }^{4}$ The current recommended daily intake for vitamin D and calcium are $400 \mathrm{lU} / \mathrm{d}$ (0-12 mo of age); $600 \mathrm{IU} / \mathrm{d}$ (1-70 years of age); and $800 \mathrm{IU} / \mathrm{d}$ for older adults ( $>70$ years of age). ${ }^{5}$ Vitamin D and calcium supplements, in combination, result in the positive periodontal condition, shallower probing depth, minimal attachment loss, and reduced alveolar bone loss. ${ }^{6,7}$

\section{Methodology}

Data was searched from the published articles of EBSCO host database and original studies regarding the effects and implications of vitamin D on oral health directly, or in an indirect manner were selected from the literature. EBSCO host is an intuitive online research platform used by thousands of institutions and millions of users worldwide. With quality databases and search features, EBSCO host helps researchers of all kinds find the information they need fast. EBSCO host offers high-quality articles licensed from reputable publishers recognized by library professionals, chosen to meet the specific needs of researchers. As per Flowchart 1, an overall, 25 original studies regarding the effect of vitamin $D$ on various parameters were screened, out of which, five studies were based on genetic basis of vitamin D, two studies were based on the effect of vitamin $D$ on dental caries, 12 studies were based on the effect of vitamin D on periodontal health and one study was based on the effect of vitamin D on the immune-compromised population.

Henceforth, the results and conclusions of some of the studies regarding this topic are:

\begin{abstract}
${ }^{1}$ Senior Lecturer, ${ }^{2}$ Associate Professor and Head, ${ }^{3}$ Ex-Principal and Head

1,2Department of Public Health Dentistry, Rayat Bahra Dental College and Hospital, Mohali, Chandigarh, India

${ }^{3}$ Department of Prosthodontics and Dental Materials, Rayat Bahra Dental College and Hospital, Mohali, Chandigarh, India
\end{abstract}

Corresponding Author: Varun Suri, Senior Lecturer, Department of Public Health Dentistry, Rayat Bahra Dental College and Hospital, Mohali, Chandigarh, India, e-mail: drvarunsuri@gmail.com

How to cite this article: Suri V, Bansal M, Kalra T. Role of Vitamin D in Dental Health: A Review. J Postgrad Med Edu Res 2019;53(2):85-88.

Source of support: Nil

Conflict of interest: None

Flowchart 1: Analysis of various studies of vitamin $D$ and dental health

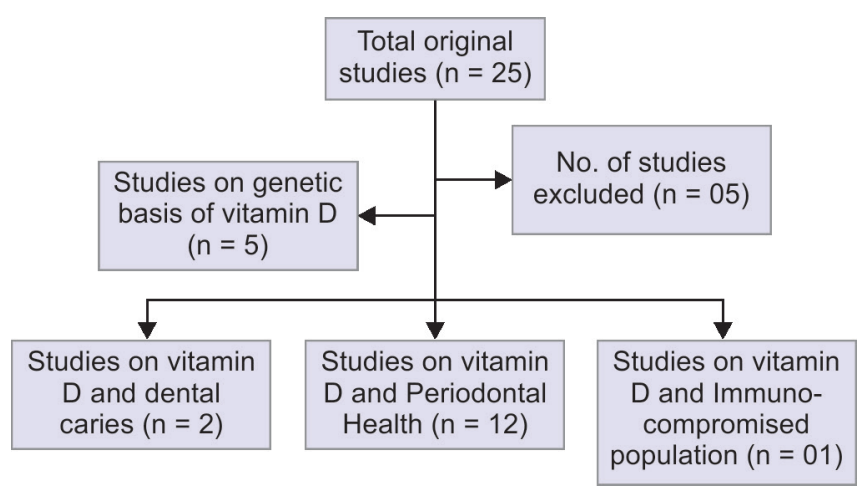

- History of vitamin D and its functions: The final active hormone derived from vitamin D was isolated and identified in 1971, and its structure was deduced as 1a,25-dihydroxyvitamin $D_{3}$ $\left[1,25(\mathrm{OH})_{2} \mathrm{D}_{3}\right]$

The basic function of vitamin $\mathrm{D}$ is to regulate normal blood levels of phosphorus and calcium. Vitamin D assists the body to absorb calcium, which regulates the structure of bones. It is used alone or together with calcium to improve bone health and

(c) The Author(s). 2019Open Access This article is distributed under the terms of the Creative Commons Attribution 4.0 International License (https://creativecommons. org/licenses/by-nc/4.0/), which permits unrestricted use, distribution, and non-commercial reproduction in any medium, provided you give appropriate credit to the original author(s) and the source, provide a link to the Creative Commons license, and indicate if changes were made. The Creative Commons Public Domain Dedication waiver (http://creativecommons.org/publicdomain/zero/1.0/) applies to the data made available in this article, unless otherwise stated. 
decrease fractures. Vitamin D acts by modifying cellular activity, cell differentiation, and cell proliferation. Vitamin D receptors are found in the immune system, intestine, pancreas, and heart, suggesting its role in these tissues. Hence, it is associated with a variety of conditions such as type 1 and type 2 diabetes, multiple sclerosis, and some cancers. Vitamin D is required throughout life. Apart from its role in bone formation, it also plays an important role in several other physiologic systems. Its use may well prevent several degenerative diseases, and it may also play a role as an anticancer agent. It also has an immunomodulatory role and its deficiency affects the T cell-mediated immunity. ${ }^{8}$

- Studies regarding the genetic basis of vitamin D: Vitamin D receptor (VDR) helps in the metabolism of vitamin D and it also plays an important role in regulating the downstream genes responsible for vitamin D activity. Yoshihara et al. in $2001^{9}$ conducted a study in a Japanese cohort of generalized early-onset periodontitis for analysis of vitamin D and Fcy polymorphisms. The hypothesis of his study was to investigate the association of genetic basis for both conditions, i.e, generalized early-onset periodontitis, and vitamin D polymorphisms. The study included 42 patients with generalized early-onset periodontitis (G-, EOP), 52 patients with adult periodontitis (AP) and 55 race-matched healthy controls $(\mathrm{HC})$. All the patients were assessed by periodontists for the number of missing teeth, probing depth (PD) and clinical attachment level (CAL). PCR technique was used for the estimation of VDR and Fcy genotypes. G-EOP patients had significantly higher mean PD and CAL compared with AP and HC groups. Distribution of VDR genotypes was almost similar in all the groups. G-EOP patients had significant over-representation of the Fcy-RIIlb genotypes compared with the AP group and $\mathrm{HC}(p=0.027$ and $p=0.006$ respectively). The study concluded that genetic polymorphisms may influence G-EOP in a complex way, though no direct relation was found in G-EOP and VDR genotype.

To understand the mechanism of action of vitamin $D$ in improving periodontal health Grenier et al. ${ }^{10}$ investigated the effect of $1,25(\mathrm{OH})_{2} \mathrm{D}_{3}$ on the growth as well as virulence factor gene expression of pathogenic bacteria, porphyromonas gingivalis. The effect of vitamin D levels on the activation of nuclear factor kappa $B$ transcription factor in monocytes was also assessed. The results showed that $1,25(\mathrm{OH})_{2} \mathrm{D}_{3}$ inhibits the gene expression for virulence factors thus attenuating the action of $P$. gingivalis. Growth of the offending bacteria was also affected by vitamin D. Nuclear factor kappa B activation was also attenuated. Author conclusion was that this knowledge can help us in finding out new therapeutic strategies and preventive modalities.

Vitamin $D$ is responsible for bone formation and calcium homeostasis. Taking this as hypothesis Ulrike et al. ${ }^{11}$ in 2016 conducted a randomized double-blind placebo-controlled trial to see whether oral supplementation with vitamin $D$ with calcium has any influence on bone formation after maxillary sinus augmentation. The second group received only calcium. It was seen that there was a significant improvement in vitamin D levels in the vitamin D group but there was no significant difference in bone formation or graft resorption between both the groups.

Sahli et al. in $2014^{12}$ conducted a study in postmenopausal women to see the association between 25(OH)D levels and concentration of oral pathogenic bacteria. Presence of Porphyromonas gingivalis, Tannerella forsythia, Fusobacterium nucleatum, Prevotellaintermedia, and Campylobacter species were assessed in the subgingival plaque by immunofluorescence method. No association was found between 25(OH)D levels and pathogenic oral bacteria.
Nociti et al. ${ }^{13}$ in their study to investigate the mechanism of action of vitamin D observed that 1,25D regulates DMP-1 expression through a VDR-dependent mechanism, which contributes to local changes in tooth mineral homeostasis.

\section{Vitamin D and Dental Caries}

Karin et al. in $2016^{14}$ conducted a study to see the effect of vitamin D on dental caries in children. It was a retrospective study, and authors used data from the National Health and Nutrition Examination Survey. The data of 5-12-year-old children were analyzed. On applying multivariate logistic regression it was observed that there was no significant association between vitamin D levels and caries in children aged $5-12$ years.

Antonenko et al. in $2015^{15}$ conducted a study in young women to see the association between oral health and calcium and Vitamin D nutritional status. The methodology consisted of recording dietary history for the intake of calcium and protein, and the serum levels of 25(OH)D were assessed. DMFT index, loesillness plaque index, and sugar intake were used to assess the dental status and caries risk. Women with high caries risk had higher DMFT index and there was a significant correlation with low calcium and low 25(OH)D levels.

\section{Vitamin D and Periodontal Health}

Zhou et al. in $2012^{16}$ conducted a case-control study to evaluate the association of $25(\mathrm{OH})$ vitamin D levels with periodontal health and chronic obstructive pulmonary disease (COPD). This study was conducted in 193 COPD patients and 181 controls. The periodontal evaluation consisted of periodontal probing, plaque index (PLI), number of remaining teeth and X-ray examination of alveolar bone. Dental caries and oral mucosa were also evaluated. The COPD group had more vitamin D deficient subjects than controls $(21.2 \%$ versus $20.4 \%$ in severe deficiency and $73.1 \%$ versus $65.7 \%$ in mild deficiency). In a linear regression analysis number of remaining teeth were significantly associated with vitamin $D$ levels in the control group ( $\mathrm{p}=0.002$ among COPD group, and $p<0.001$ among control group). On multivariate analysis, it was found that there was a significant correlation between lower vitamin D levels and poor indices only in COPD patients who were former smokers. Though retrospective, this study shows a positive association between vitamin D status and periodontal health.

Alshouibi et al. in $2013^{17}$ conducted a study to see the association between vitamin $D$ and periodontal health in older men. Study participants were selected from adult males who were examined under the Department of Veterans Affairs Dental Longitudinal study (VADLS). Five hundred and sixtytwo members with a mean age of 62.9 years were examined 1-4 times between 1986 and 1998, over a period of 12 years. The periodontal examination consisted of calibrated examiner measured probing pocket depth (PPD) and attachment Loss (AL) on each tooth. Radiographs were used for determining alveolar bone loss (ABL). Severe clinical periodontal disease was defined as at least 1 site with PPD $\geq 5 \mathrm{~mm}$ and 2 or more sites with $\mathrm{AL}$ $\geq 6 \mathrm{~mm}$, not on the same tooth. Moderate to severe $A B L$ was defined as at least 3 proximal sites (i.e., $\geq 2$ teeth) with $40 \%$ or more bone loss. A standard questionnaire was used to calculate total nutrient intake. Three cut off points for a dose of vitamin $D$ were used. These were daily intakes of less than $400 \mathrm{IU}$, equal to or more than $400 \mathrm{IU}$ but less than $800 \mathrm{IU}$, and $800 \mathrm{IU}$ or more. These cut-off points were based on the dietary recommendations from the International Osteoporosis Foundation (IOF) and the Institute of Medicine (IOM). Results showed that total vitamin D 
intake was inversely associated with odds of severe periodontitis and moderate to severe $A B L$ after adjusting for demographic and other confounding parameters. The odds of severe periodontitis among men consuming $\geq 800 \mathrm{IU} /$ day were $0.67(95 \% \mathrm{Cl}, 0.55-0.81)$ relative to those of men consuming $<400 \mathrm{IU} /$ day.On further analysis, it was observed that Each $100 \mathrm{IU}$ increment in daily total vitamin $D$ intake was independently associated with reduced odds of severe periodontal disease $(\mathrm{OR}=0.97,95 \% \mathrm{Cl}=0.96-0.98)$ and moderate-to-severe $\mathrm{ABL}(\mathrm{OR}=0.95,95 \% \mathrm{Cl}=0.91-0.99)$. The authors concluded that the maintenance of vitamin D intake near or above the recommendations could be a safe and effective method of reducing periodontal disease prevalence. This can be done either by improving the nutrition or giving supplementary doses of vitamin $\mathrm{D}$.

Hiremath et al. ${ }^{18}$ conducted a dose-response randomized control trial in 2013 to assess the effect of vitamin D on gingival health. The basis of this study was to see the anti-inflammatory effect of various doses of vitamin $D$ in patients with gingivitis. The study was conducted in Belgaum India. Males and females aged 18-64 years diagnosed with different stages of gingivitis were recruited for the study. The other inclusion criterion was the serum levels of 25 hydroxyvitamin D to range between 20-65 ng/ $\mathrm{ml}$ at the baseline. Ninety-six recruited subjects were randomized in four groups. Group A subjects received vitamin D in a dose of 2000 IU per day, group B subjects received 1000 IU of vitamin D per day, group C subjects were given 500 IU of vitamin D daily and the fourth group $D$ were given placebo. The follow-up visits were at 30, 60 and 90 days. It was observed that there was a significant change in the gingival score in all the interventional groups except placebo group and the response was directly proportional to the dose of vitamin D. Significant anti-inflammatory effect was seen in group $A$ at one month, group $B$ at 2 months and 3 months in group $C$ $(p<0.0001)$. This study has a lot of limitations. The details of treatment given to the patients and the effect of age on the response have not been mentioned.

Schroth et al. in $2016^{19}$ conducted a study to assess the relationship between vitamin D status and dental caries in school children participating in the Canadian Health Measures Survey (CHMS). It was a cross-sectional survey of children 6-11 years of age. The methodology included a questionnaire and dental examination. Twelve calibrated dental physicians completed the dental examinations. Presence or absence of caries and DMFT index were the two outcome variables. Vitamin D status was determined by measuring serum concentrations of $25(\mathrm{OH})$ vitamin D. Levels of $>75 \mathrm{nmol} / \mathrm{L}$ were taken as optimal. More than 1000 students participated in the study. Fifty-six percent of them had evidence of caries. The DMFT score was 2.47. 87.5\% of children had vitamin D concentration $>50 \mathrm{nmol} / \mathrm{L}$ and $49.7 \%$ had optimal concentrations. Children with optimal concentrations of vitamin $D$ had significantly lower odds for caries $(\mathrm{OR}=0.57,95 \% \mathrm{Cl} 0.39$ to 0.82 ). On applying the logistic analysis it was observed that $25(\mathrm{OH}) \mathrm{D}$ levels $\geq 50 \mathrm{nmol} / \mathrm{L}$ were significantly and independently associated with lower adjusted odds for caries $(\mathrm{OR}=0.46,95 \% \mathrm{Cl}$ 0.26 to 0.83 ).

Chantarangsu et al. in $2016^{20}$ conducted a cross-sectional study in Thai population to see the relationship between chronic periodontitis (CP) and vitamin D polymorphism. 1460 patients aged 39-66 years were recruited. Vitamin D polymorphism was estimated by real-time PCR technique. Patients were divided into three groups: mild, moderate and severe chronic periodontitis. Severe CP was associated with Fokl polymorphism with an odds ratio of 1.9. The combination of being a smoker and having polymorphism increased the risk of CP (OR 9.6). Smoking and vitamin D polymorphism had a synergistic effect.

In another study by Orlando et al. ${ }^{21}$ in 2016, the association between vitamin D levels and chronic periodontitis was evaluated in Puerto Rican adults. It was a case-control study where 24 adults with moderate and severe periodontitis were compared with an equal number of healthy controls. They were matched for age, sex, and BMI. A complete periodontal examination was carried out and serum levels of $25(\mathrm{OH})$ D levels were estimated. Results showed significantly lower mean serum levels of vitamin $D$ in cases $(18.5+$ $4.6 \mathrm{ng} / \mathrm{mL})$ than in controls $(24.2+7.1 \mathrm{ng} / \mathrm{mL})$ with $p$ value of 0.006 . Odds of having periodontal disease per unit of vitamin $D$ level were lower (OR 0.885;95\% Cl 0.785, 0.997; $p<0.05$ ).

Sonja et al. in $2016^{22}$ conducted a study to see the association between tooth loss and serum concentrations of 25 hydroxyvitamin D levels in a cohort of postmenopausal women. At baseline number of missing teeth was noted and 5-year tooth loss was recorded at follow up. After applying logistic regression it was observed that there was no association between history of tooth loss and vitamin D status.

Raza et al. ${ }^{23}$ conducted a randomized controlled trial to see the effect of vitamin D supplementation during pregnancy on the periodontal health of the mother and birth weight of the baby. This study was carried out in 85 Pakistani women during early pregnancy. Periodontal examination was carried out and periodontal probing depth (PD), bleeding on probing (BOP) and attachment loss (AL) were assessed. Serum vitamin D levels were measured. Birth weights of the neonates were recorded. On analysis, it was seen that there was no difference in birth weight and periodontal status when compared between both groups.

Lee et al. in $2015^{24}$ conducted a cross-sectional study to see the association between vitamin $\mathrm{D}$ status and periodontitis. More than 6000 subjects participating in the Korea National Health and Nutrition Examination Survey were examined. Serum vitamin D levels were assessed and deficiency was defined as levels $<20 \mathrm{ng} /$ $\mathrm{mL}$. Community periodontal index (CPI) was used as a parameter to define periodontal health. On analysis, it was found that there was no association between vitamin D levels and periodontitis. Some association was seen only in current smokers.

Antonoglou et al. in $2015^{25}$ conducted a study to see the protective effect of vitamin D on periodontal health by measuring pocketing and gingival bleeding. Subjects were selected from the Finnish Health 2000 survey. Serum levels were assessed for $25(\mathrm{OH})$ $D$ and periodontal examination consisted of measuring periodontal pockets and looking for bleeding sextants. There was no association between vitamin D levels and teeth with deep periodontal pockets and the number of bleeding sextants thus negating the role of vitamin $\mathrm{D}$ in periodontal health.

In Buffalo, Osteo Perio study by Millen et al. ${ }^{26}$ association between $25(\mathrm{OH})$ D levels and 5 years follow-up status of periodontal health in postmenopausal women was investigated. The parameters for oral health used included, alveolar crest height, clinical attachment level, probing depth and percentage of gingival sites that bled on assessment. There was no association between vitamin $\mathrm{D}$ status and subsequent 5 -year change in periodontal disease measures.

Millen et al. in $2013^{27}$ conducted a cross-sectional study in 900 postmenopausal women to assess the association between $25(\mathrm{OH})$ $\mathrm{D}$ concentration and periodontal disease. Indicators of chronic disease were alveolar crestal height $(\mathrm{ACH})$, tooth loss, clinical attachment level and probing depth (CDC and AAP criteria). Acute inflammation was assessed by gingival bleeding sites. Vitamin 
D levels were defined as adequate $25(\mathrm{OH}) \mathrm{D}>50 \mathrm{ng} / \mathrm{mL}$ ) and inadequate $25(\mathrm{OH}) \mathrm{D}<50 \mathrm{ng} / \mathrm{mL}$ ). There was no association between vitamin D levels and periodontal disease defined by $\mathrm{ACH}$ and tooth loss. On the other hand women with adequate vitamin $D$ levels had 33\% lower odds of having chronic periodontal disease as per CDC/AAP criteria when compared to inadequate vitamin D status. Women with adequate vitamin D had $42 \%$ lower odds of having $>50 \%$ of bleeding gingival sites thus proving the role of vitamin D as anti-inflammatory or immune-modulator.

\section{Vitamin D in Immunecompromised Population}

In a group of immunecompromised person effect of vitamin D on oral health may be different. In such a study by Sroussi et al. ${ }^{28}$ in 2012, the relationship between oral candidiasis and vitamin D levels in HIV positive women was studied. A subset of women participating in women's interagency HIV study (WIHS) was further examined for evidence of oral candidiasis. Serum $25(\mathrm{OH})$ vitamin D levels were measured in 84 Chicago HIV positive women. Fourteen $(16,7 \%)$ were diagnosed with oral candidiasis. Fifteen women were vitamin D sufficient, 23 were vitamin D insufficient and 34 women were deficient in vitamin $\mathrm{D}$. The prevalence rate of oral candidiasis was maximum (26.1\%) in women with vitamin D deficiency which was significantly high as compared to other two groups $(p<0.05)$. On multivariate analysis of other associated factors for oral candidiasis, vitamin D deficiency emerged as a significant predictor of oral candidiasis with an adjusted odds ratio of 5.66 [95\% confidence interval $(\mathrm{Cl}) 1.01-31.71](p<0.05)$. This study only found an association between vitamin D levels and OC, whether it is the cause can only be clarified by further prospective interventional studies.

Hence, the studies based on the relationship of periodontal health and vitamin D depict an overall positive impact of vitamin $D$ in maintaining healthy periodontium.

\section{Conclusion}

Vitamin D plays a vital role in the overall maintenance of oral health. Literature is replete with studies of vitamin $D$ and its association with oral health, but there is a paucity of randomized controlled trials regarding the effect of vitamin $D$ in the unhealthy states.

\section{References}

1. Gorham ED, Garland CF, Garland FC, et al. Optimal Vitamin D status for colorectal cancer prevention: A quantitative meta analysis. AmJ Prev Med 2007;32(3):210-216.

2. Skinner HG, Michaud DS, Giovannucci E, et al. Vitamin D intake and the risk for pancreatic caneer in two cohort studies. Cancer Epidemiol Biomarkers Prev 2006;15(9):1688-1695.

3. Giovannucci E, Liu Y, Rimm EB, et al. Prospective study of predictors of Vitamin D status and cancer incidence and mortality in men. J Natl Cancer Inst 2006;98(7):451-459.

4. Moore C, Murphy MM, Keast DR, et al. Vitamin D Intake in the United States. J Am Diet Assoc 2004;104(6):980-983.

5. Ross AC, Taylor CL, Yaktine AL, et al. Dietary References Intakes for Calcium and Vitamin D. Washington (DC); National Academies Press (US): 2011.

6. Miley DD, Garcia MN, Hildebolt CF, et al. Cross-sectional study of Vitamin D and calcium supplementation effects on chronic periodontitis. J Periodontol 2009;80(9):1433-1439.

7. Garcia MN, Hildebolt CF, Miley DD, et al. One-year effects of Vitamin D and calcium supplementation on chronic periodontitis. J Periodontol 2011;82(1):25-32.
8. Deluca HF. Overview of general physiologic features and functions of vitamin D. Am J Clin Nutr 2004;80(6):1689s-1696s.

9. Yoshihara A, Sugita N, Yamamoto K, et al. Analysis of Vitamin D and FcY Receptor polymorphisms in Japanese patients with Generalized Early Onset Periodontitis. J Dent Res 2001;80(12):2051-2054.

10. Grenier D; Morin MP, Fournier-LJ, et al. VitaminD inhibits the growth of and virulence factor gene expression by Porphyromonasgingivalis and blocks activation of the nuclear factor kappa B transcription factor in monocytes. J Periodontal Res 2016;51(3):359-365.

11. Schulze-SU, Dietrich T, Wu C, et al. Systemic vitamin D supplementation and local bone formation after maxillary sinus augmentation-a randomized, double-blind, placebo-controlled clinical investigation. Clin Oral Implants Res 2016;27(6):701-706.

12. Sahli MW, Wactawski-Wende J, Ram PK, et al. Association of Plasma 25 -Hydroxyvitamin $D$ concentrations and pathogenic oral bacteria in postmenopausal females.J Periodontol 2014;85(7):944-955.

13. Nociti FHJr, Foster BL, Tran AB, et al. VitaminD represses dentin matrix protein 1 in cementoblasts and osteocytes. J Dent Res 2014;93(2): 148154.

14. Herzog K, Scott JM, Hujoel P, et al. Association of VitaminD and dental caries in children: Findings from the National Health and Nutrition examination survey, 2005-2006. J Am Dent Assoc 2016;147(6): 413-420.

15. Antonenko O, Bryk G, Brito G, et al. Oral health in young women having a low calcium and Vitamin D nutritional status. Clin Oral Investig 2015;19(6):1199-1206.

16. Zhou X, Han J, Song Y, et al. Serum levels of 25 -hydroxy vitamin D, oral health and chronic obstructive pulmonary disease. J ClinPeriodontol 2012;39(4):350-356.

17. Alshouibi EN, Kaye EK, Cabral HJ, et al. Vitamin D and periodontal health in older men. J Dent Res 2013;92(8):689-693.

18. Hiremath VP, Bhasker Rao CB, Naik V, et al. Anti inflammatory effect of Vitamin D on gingivitis: A dose-response randomized control trial. Oral Health Prev Dent 2013;11:61-69.

19. Schroth RJ, Rabban R, Loewen G, et al. Vitamin D and dental caries in children. J Dent Res 2016;95(2):173-179.

20. Chantarangsu S, Sura T, Mongkornkarn S, Donsakul K, et al. Vitamin Dreceptor gene polymorphism and smoking in the risk of chronic periodontitis. J Periodontol 2016;87(111):13431.

21. Abreu OJ, Tatakis DN, Elias-Boneta AR, et al. Low Vitamin D status strongly associated with periodontitis in Puerto Rican adults. BMC Oral Health 2016;16(1):89.

22. Pavlesen S, Mai X, Wactawski-Wende J, et al. VitaminDstatus and tooth loss in postmenopausal females: The buffalo osteoporosis and periodontal disease (Osteo Perio) study. J Periodontol 2016;87(8): 852-863.

23. Khan FR, Ahmad T, Hussain R, et al. A randomized controlled trial of Oral Vitamin $D$ supplementation in pregnancy to improve maternal Periodontal health and birth weight. Journal of International Oral Health 2016;8(6):657-665.

24. Lee HJ, Je DI, Won SJ, et al. Association between Vitamin D deficiency and periodontal status in current smokers. Community DentOralEpidemiol 2015;43(5):471-478.

25. Antonoglou, GN, Suominen AL, Knuuttila M, et al. Associations between serum 25-hydroxyvitamin $D$ and periodontal pocketing and gingival bleeding: results of a study in a non-smoking population in Finland. J Periodontol 2015;86(6):755-765.

26. Millen AE, Andrews CA, LaMonte MJ, et al. VitaminD Status and 5-year changes in Periodontal Disease measures among postmenopausal women: the Buffalo OsteoPerio Study. J Periodontol 2014;85(10): 1321-1332.

27. Millen AE, Hovey KM, LaMonte MJ, et al. Plasma 25 -Hydroxyvitamin D concentrations and periodontal disease in postmenopausal women. J Periodontol 2013;84(9):1243-1256.

28. Sroussi HY, Miller JB, French AL, et al. Association among Vitamin D, oral candidiasis and calprotectinemia in HIV. J Dent Res 2012;91(7): 666-670. 